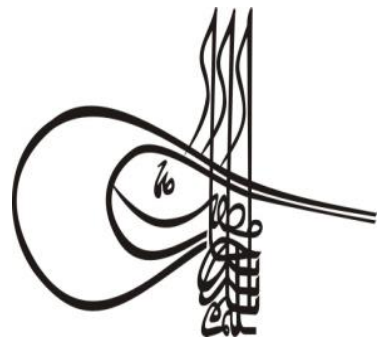

Received/Geliş: 28.06 .2019

\section{Turkígl Studieg}

Volume 14 Issue 4, 2019, p. 2833-2843

DOI: 10.29228/TurkishStudies.23458

ISSN: $1308-2140$

Skopje/MACEDONIA-Ankara/TURKEY

Research Article / Araștırma Makalesi

Article Info/Makale Bilgisi

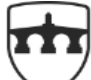

INTERNATIONAL BALKAN UNIVERSITY

EXCELLENCE FOR THE FUTURE IBU.EDU.MK

$\checkmark$ Accepted/Kabul: 10.08 .2019

Go Report Dates/Rapor Tarihleri: Referee 1 (25.07.2019)-Referee 2 (25.07.2019)

This article was checked by iThenticate.

\title{
NOMINALKOMPOSITA IM TÜRKISCHEN UND DEUTSCHEN
}

\author{
Ayşe UYANIK*
}

\section{ZUSAMMENFASSUNG}

Nominalkomposita zählen $\mathrm{zu}$ den meist gebrauchten Wortbildungen der deutschen Sprache. Sie sind Kompositionen deren Glieder mindestens aus zwei Nomen entstehen. Sie existieren auch in der türkischen Sprache, jedoch ihre Anzahl ist unterlegen und Bildung, unterschiedlich. Da die DaF- Lernenden diese Abweichungen und Gemeinsamkeiten der Wortbildungsprozesse von den erwähnten Sprachpaaren nicht beherrschen bevorzugen sie andere Bildungen und Äußerungsmöglichkeiten, die eigentlich ein fließendes Deutsch hindern. Dagegen entsprechen den unvollständigen Genitiven im Türkischen, die gleichzeitig auch als Komposita gelten aber getrennt geschrieben werden, deutsche Nominalkomposita. Doch die DaF- Lerner sind über diese Entsprechung nicht informiert. Diese Arbeit beabsichtigt den DaF Lernenden die Übersetzungsmöglichkeit der türkischen unvollständigen Genitivkonstruktionen durch Nominalkomposita ins Deutsche zu vorschlagen. Dafür wird im theoretischen Teil die Nominalkomposita im Türkischen und Deutschen vorgeführt. Nachdem wird mit einer Übersetzungsaufgabe versucht festzustellen, wie sie unvollständige Genitive aus dem Türkischen ins Deutsche übersetzen. An dieser Durchführung kann gesehen werden, dass die Lernenden in diesem Bereich kenntnislos sind. Sie sind der Zusammensetzung von Nomen genau so fremd wie den Fugenelementen, die für die Naht dieser Nominalkomposita notwendig sind. Wenn die DaF- Lernenden mit diesen Wortbildungsprozessen bekannt gemacht und diese Konstruktionen ihnen beigebracht werden, können sie sich in der Zielsprache viel besser ausdrücken und über eine hochwertigere Sprachkompetenz verfügen.

Schlüsselwörter: Komposita, Nominalkomposita, unvollständige Genitivkonstruktionen, Wortbildung 


\title{
NOMINAL COMPOUNDS IN TURKISH AND GERMAN
}

\begin{abstract}
NOMINAL compounds are among the most commonly used word formations in the German language. They are compositions whose members arise from at least two nouns. They also exist in the Turkish language, but their numbers are inferior and education varies. Since the students do not master these deviations and similarities of word formation processes from the mentioned language pairs, they prefer other formations and utterances that actually prevent a fluent German. In contrast, the incomplete genitives in Turkish correspond to German nominal compounds. But the GFL learners are not informed about this correspondence.This thesis intends to propose to GFL learners the possibility of translation of Turkish incomplete genitive constructions by nominal composite into German For this purpose, the nominal components in Turkish and German are presented in the theoretical part. After that, a translation task tries to pin down how to translate incomplete genitives from Turkish into German. In this implementation can be seen that the learners in this area are ignorant. They are as alien to the composition of nouns as the joint elements necessary for the suture of these nominal compounds. When the DaF learners are introduced to these word formation processes and are taught these constructions, they can express themselves much better in the target language and have a higher level of language proficiency.
\end{abstract}

\section{STRUCTURED ABSTRACT}

Turkish and German are largely differentiated by agglutination and inflection. Since they come from different language families, besides genealogical differences, there are also notable differences between syntax and morphology, especially word formation. The essential word formation processes in German are derivation and composition.As an agglutinating language, the derivation is mainly known through the Suffigieren, also the Turkish language, but the composition follows different and does not occur as often as in German.The role of the composition for the German vocabulary should not be underestimated."The composition makes the biggest contribution to this, especially the nominal composition, which accounts for around two thirds of the vocabulary (Duden, 1995, p. 399)".In the same way, the composition also builds vocabulary in Turkish.However, the "number of Komposita in Turkish is compared to German very low" and different. "Instead of composites, genitive constructions are favored in which the attributive genitive loses its case suffix (Balc1, 2009, p. 133)".Because of this, the GFL learners in Turkey are unfamiliar with the word formation of their native language and mainly have problems in teaching translation of the source language into the target language. If for example, a translation of "araba kapısı" is required, they often write "die Tür des Autos" or they translate every word for themselves, writing separately "Auto Tür" instead of a composition: Autotür. Because the counterparts of these translations to some extent coincide in meaning, they lead the GFL learners to erroneous transmissions. Because they differ 
semantically and grammatically in the target language. "The door of the car" in Turkish means "araba nin kapısı", which is a complete genitive construction in Turkish and can be translated in German by a genitive. Both nouns have a suffix. But because "araba kapisı" loses its genitive suffix, a translation would be more legitimate as a composite. Although according to the Turkish grammar, words such as "araba kapisı" are suitable as an incomplete genitive construction for compositions, a composition does not catch the learners. This mistake makes it difficult for German learners to come up with the idea of composition. Because they are not accustomed to their native language compound words and usually prefer the composition "incomplete genitive - groups are written separately (Röhrborn, 1990, p. 59)".On the other hand, German allows the seams of the individual words through so-called joint elements which, for better knowledge of German, offer a single word as a grammatical building block (Balc1, 2015, p.76) instead of a multi-linked character string, or a compound. The aim of this work is to show the students the possibility of translating the Turkish incomplete genitive constructions by nominal composites into German. Thus, the learners of German approach the word formation by composition in German and afford them a better command of this language. In the first part of the thesis, the nominal composition was theoretically investigated in Turkish and German. First, an exercise was performed with them. In order to determine which educational mistakes German learners can make during a composition demanding translation, a simple translation task was asked. The tasks were carried out by 93 students of the Department of German Language and Literature from the first and second grade of the Selçuk University. They asked for a translation from Turkish into German. They should translate ten selected sentences, the incomplete genitive, whose German translation corresponds to a compound. Thus, the observation of their composition errors was achieved for a compound in German. For this reason, initially incomplete genitives that are formed in German as composites without joint elements but have to be written together have been demonstrated. Next, again 6 incomplete genitives selected in German as Komposita, the common Fugenelemente "-e, - (e) s, - (e) n, -er". Accordingly, the German learners should write together the first incomplete genitives yaz yağmuru, sonbahar güneşi, oturma odası, and uçak bileti Fugenlos. Whereas the next ones definitely need a joint. In the example sentences, the most common joint elements were intentionally preferred. It has been seen that they are ignorant of the nomenclature of nominal compounds, and that these reasons discourage them from composing the nominal compounds.

- Since they are foreign to the composition of their mother tongue, the DaF learners generally prefer the separate writing of the individual nouns. Instead of nominal compounds, they prefer either the complete genitive or long consecutive words or phrases.- Because they have no idea about the word formation process of the language pair, they can not face the formation of the compound.

- You have no knowledge of joint elements that provide the seam of the German Nominalkomposita. That is why they write the incomplete genitives except they are already well known, often separated, if already together without joint elements. 
- They donate the Nominalkomposita in Turkish a membership, by making a possessive genitive but actually make a grammatical and semantic error transfer.

- The importance of these compounds for the German vocabulary is not known to them.

Keywords: compounds, nominal compounds, incomplete genitive constructions, word formation

\section{Einleitung}

Das Türkische und Deutsche unterscheiden sich größtenteils durch Agglutination und Flexion. Da sie aus unterschiedlichen Sprachfamilien stammen, sind außer genealogischen Unterschieden, auch bemerkenswerte Abweichungen zwischen der Syntax und Morphologie, besonders der Wortbildung. Die wesentlichen Wortbildungsprozesse im Deutschen sind Derivation und Komposition. Als eine agglutinierende Sprache ist die Derivation vornehmlich durch das Suffigieren, auch der türkischen Sprache sehr bekannt, doch die Komposition folgt anders und kommt nicht so oft wie im Deutschen vor. Die Rolle der Komposition für den deutschen Wortschatz ist nicht zu unterschätzen. „Den größten Beitrag dazu leistet die Zusammensetzung, darunter vor allem die Nominalkomposition, der rund zwei Drittel des Wortschatzes zu verdanken sind (Duden, 1995, S. 399)“. In gleicher Weise baut die Komposition auch im Türkischen den Wortschatz an. Jedoch die „Anzahl der Komposita im Türkischen ist im Vergleich zum Deutschen sehr gering” und unterschiedlich. „Statt Komposita werden Genitivkonstruktionen favorisiert, in denen der attributive Genitiv sein Kasussuffix verliert (Balc1, 2009, S. 133)". Deswegen sind die DaF- Lerner in der Türkei dieser Wortbildung ihrer Muttersprache her fremd und weisen hauptsächlich Probleme im Übersetzungsunterricht von der Ausgangssprache in die Zielsprache auf.

Da es in der universitären Stufe angenommen wird, dass das Regelsystem des Türkischen den Lernenden von der Schule schon bekannt ist, wird nur die Grammatik des Deutschen unterrichtet. In den Grammatikbüchern wird die Wortbildung schon behandelt, jedoch die Komposition allein nicht. Wieder im Übersetzungsunterricht kommt es bei den Übersetzungsübungen, anstatt einer grammatischen Didaktik, noch mehr einer ganz geringen kontrastiven Gegenüberstellung der Wortschätze und Sprachstrukturen. Doch „für den Übersetzer ist zweifellos die Kenntnis aller produktiven Wortbildungsmechanismen von Bedeutung (Weber, 2016, S. 36)”. Deswegen müssen sie die Gemeinsamkeiten und Unterschiede des Sprachpaares selbst feststellen und interpretieren. Leider gelingt ihnen ein wirksamer Vergleich oftmals nicht. Denn manche Strukturen gleichen sich, entsprechen ihrer Übersetzung, aber ihr Gebilde in der Gegensprache ist unterschiedlich. Wenn z. B. eine Übersetzung von „araba kapısı“ verlangt wird schreiben sie öfters „die Tür des Autos“ oder sie übersetzen jedes Wort für sich, schreiben getrennt „Auto Tür“ anstatt einer Zusammensetzung: Autotür. Weil die Gegenentsprechungen dieser Übersetzungen sich der Bedeutung her einigermaßen decken, führen sie die DaF- Lerner zu Fehlübertragungen. Denn sie unterscheiden sich in der Zielsprache semantisch sowie grammatikalisch. „Die Tür des Autos“ bedeutet auf Türkisch „,araba-nın kapısı“, das eine vollständige Genitivkonstruktion im Türkischen ist und im Deutschen durch einen Genitiv übersetz werden kann. Beide Nomen haben ein Suffix. Aber weil bei „araba kapısı“ das Bestimmungswort das Genitivsuffix verliert wäre eine Übersetzung als Kompositum rechtmäßiger. Obwohl nach der türkischen Grammatik Wörter wie „araba kapısı“ als unvollständige Genitivkonstruktion für Zusammensetzungen geeignet zählen, fällt den Lernenden eine Zusammensetzung nicht ein. Dieser Missgriff erschwert den Deutschlernern den Einfall von Komposition. Denn sie sind ihrer Muttersprache her den zusammengesetzten Wörtern nicht gewöhnt und bevorzugen üblicherweise die Zusammensetzung ,unvollständige Genitiv - Gruppen die getrennt geschrieben werden (Röhrborn, 
1990, S. 59)". Hingegen ermöglicht Deutsch die Naht der einzelnen Wörter durch sogenannte Fugenelemente, die für bessere Deutschkenntnisse „statt einer mehrgliedrigen Zeichenkette ein einziges Wort als grammatischen Baustein (Balc1, 2015,S.76)", bezieungsweise ein Kompositum bieten.

Ziel dieser Arbeit ist es, den $\mathrm{DaF}$ - Lernenden die Möglichkeit der Übersetzung der türkischen unvollständigen Genitivkonstruktionen durch Nominalkomposita ins Deutsche zu zeigen. Somit den Deutschlernern die Wortbildung durch Komposition in Deutsch annähern und ihnen eine bessere Beherrschung dieser Sprache leisten. Denn schon in Fremdsprachen aus der gleichen Sprachfamilie zeigt ihre Übersetzung Schwierigkeiten. „Viele deutsche Komposita können nur durch Wortgruppen in durchaus nah verwandten Sprachen wie das Englische und Französische übertragen werden (Graefen \& Liedke, 2012, S. 99)“. Deswegen möchten wir im Weiteren ein durchgeführtes Unterrichtsbeispiel, in denen besonders unvollständige Genitive aus dem Türkischen, die ins Deutsche mit und ohne Fugenelemente übersetzt und zusammengeschrieben werden vorlegen und Alternativen vorschlagen.

\section{Nominalkomposition im Türkischen}

Die Nominalkomposition im Türkischen findet durch die Zusammensetzung von zwei oder mehreren Substantiven, die einen neuen Begriff bilden, statt. Es handelt sich immer um die Bezeichnung eines Gegenstandes oder Wesens durch eine Wortgruppe. Beispielsweise Ayakkabı, baş ağrısı, kol saati, gece kondu, hanımeli usw. Aber eine jeweilige feste Zusammenschreibung wie im Deutschen ist nicht zu sehen. Ganz im Gegenteil ihre Rechtschreibung ist eine lang umstrittene Diskussion. Ob sie zusammen oder getrennt geschrieben werden soll, zeigt schon seit der Schriftreform 1928 keine Übereinstimmung. Sie hat heute noch keine festen Regeln (Zülfikar, 2013). Beispielsweise können Komposita wie „dilbilim/dilbilim“ (Sprachwissenschaft), anadil/anadil (Muttersprache) oder "haftasonu/haftasonu" (Wochenende) zusammen oder getrennt geschrieben werden. In der neu erschienenen Rechtschreibung (TDK Yazım Klavuzu 2012) sind sie zu meist als Komposita getrennt geschrieben. Aber getrennt geschriebene Nominalkomposita bilden im Türkischen gleichzeitig auch unvollständige Genitivkonstruktionen. Diese Obskurität der Muttersprache erschwert den Deutschlernenden die Entscheidung für eine Komposition im Deutschen. Denn die Komposita haben im Türkischen nicht den gleichen Stellenwert wie im Deutschen. Größtenteils sind unvollständige Genitivkonstruktionen die Wortgruppe, die sich für Zusammensetzung eignen (Korkmaz, 2009, S. 139; Özkan, 2018, S. 2; Ergin, 2009, S. 385). Zwar ermöglicht Türkisch „durch Genitivkonstruktion und adjektivische Fügungen die Komposition“" auch (Uslu, 2016, S. 68), doch öfters nicht wie Deutsch durch Zusammensetzung, sondern Einzelschreibung. Was im Türkischen ,als ein Satz oder als ein Syntagma erscheint, kommt im Deutschen als Komposition vor (Balc1, 2015, S. 77)". Zum Beispiel übersetzt man eine Wortgruppe wie „yabanc1 dil dersi“, genügt allein das Wort „Fremdsprachenunterricht“ oder wird eine Wortgruppe, wie „yabancı dil bilen büro elemanı“ ins Deutsche übertragen, reicht das aus drei Komponenten entstehende, mit einem Fugenelement vereinte einzige Wort „Fremdsprachenkorrespondent“ aus.

\section{Nominalkomposition im Deutschen}

In Duden Grammatik werden „Wörter, die ohne zusätzliche Ableitungsmittel aus zwei oder mehreren selbstständig vorkommenden Wörtern gebildet sind“" als Komposition definiert. Kompositionen ,deren Glieder aus Nomina bestehen (Duden, 1982, S. 527) ,werden als Nominalkomposition oder Substantivkomposita bezeichnet. Sie setzen sich mindestens aus zwei Konstituenten. Das an erster Stelle stehende Wort wird als Bestimmungswort; und an zweiter Stelle stehende als Grundwort bezeichnet. In dem Kompositum „Apfelbaum“ ist das Wort „Apfel“ das Bestimmungswort und „Baum“ das Grundwort. Doch nicht immer ist diese einfache Zusammensetzungsweise anzuwenden. Wenn z. B. von einem Baum der eine Tanne ist, ein Kompositum gebildet wird, heißt es „Tannenbaum“. Denn öfters fördert ihre Zusammensetzung ein Bindemittel. Diese „Nahtstelle zwischen den Konstituenten einer komplexen Wortbildung wird als Fuge 
bezeichnet" (Fleischer \& Barz, 2012, S. 185). Deswegen werden sie in morphologischer Hinsicht in zwei Typen unterschieden: Komposita ohne Fugenelemente und Komposita mit Fugenelemente (vgl. (Kahramantürk, 1998, S. 163)". Die zwischen den Konstituenten eingeschobenen Fugen können je nach der Aufforderung des Wortes „,-(e)s, -(e)n, -e, -er, -ens“ lauten. "Etwa ein Drittel aller deutschen Komposita weisen ein Fugenelement auf. Insbesondere in N+N- Komposita sind sie häufig anzutreffen (Varsami, 2008)“.Wenn sie auch wie Plural- oder Genitivendungen aussehen und eigentlich sprachgeschichtlich aus diesem entstanden sind (vgl. Wegener, 2006, Elshorbagy, 2007, Schlücker, 2012) zeigt ihre Bildung immer Unterschiede und hat keine feste Regelung. Auch diese Regelungslose Bildungsspanne erschwert den Fremdsprachenlernern die Übersetzung der unvollständigen Genitive aus dem Türkischen in die Deutschen Kompositums.

\section{Fehlgriff der Lernenden bei den Übungen}

Um feststellen zu können welche Bildungsfehler die Deutschlernenden während eine Komposition fordernde Übersetzung machen können, wurde ihnen eine einfache Übersetzungsaufgabe gestellt. Die Aufgaben wurden von 93 Studenten der Abteilung deutsche Sprache und Literatur aus der ersten und zweiten Klasse von der Selçuk Universität ausgeübt. Es wurde von ihnen eine Übersetzung von Türkisch ins Deutsche verlangt. Sie sollten zehn ausgesuchte Sätze, die unvollständige Genitive, dessen deutsche Übersetzung einem Kompositum entspricht, übersetzen. Damit wurde die Beobachtung ihrer Fehler bei der Zusammensetzung für ein Kompositum im Deutschen erzielt. Deswegen wurden anfänglich 4 unvollständige Genitive die im Deutschen als Komposita ohne Fugenelement gebildet aber zusammengeschrieben werden müssen vorgeführt. Nächstens wieder 6 unvollständige Genitive die im Deutschen als Komposita, die die geläufigen Fugenelemente ,-e, -(e)s, -(e)n, -er“ fordern ausgewählt. Demnach sollten die Deutschlernenden die ersten unvollständigen Genitive yaz yağmuru, sonbahar güneşi, oturma odası, und uçak bileti fugenlos zusammenschreiben. Wogegen die nächsten unbedingt eine Fuge benötigen. In den Beispielsätzen wurden absichtlich die meist vorkommenden Fugenelemente bevorzugt. So dass die Lernenden erst den Ablauf der Komposition mitbekommen.

1. Yaz yağmuru çabuk geçer.

2. Penceredeki çiceği sonbahar güneşiı sitıyordu.

3. Her evde bir oturma odası bulunur.

4. İstanbul için iki uçak bileti aldım.

5. Masanın üzerinde bir çikolata kutusu duruyordu.

6. Gece yarısı kapıçaldı.

7. Öğle arası arkadaşlarla buluştuk.

8. Tarih öğretmeni II. Dünya Savaşını anlattı.

9. Bu hastanede çocuk doktoru yok.

10.En sevdiğim yemek Mercimek çorbası.

Die erwarteten Übersetzungen der unvollständigen Genitive aus dem Türkischen sollten im Deutschen wie in der Tabelle übersetzt werden: 
Tablo 1: Nominalkomposita mit und ohne Fugenelemente

\begin{tabular}{|ll|ll|}
\hline \multicolumn{2}{|c|}{ Komposita ohne Fugenelement } & \multicolumn{2}{c|}{ Komposita mit Fugenelement } \\
\hline Yaz yağmuru & Sommerregen & Gece yarısı & Mitt - er-nacht \\
\hline Sonbahar güneşi & Herbstsonne & Öğle arası & Mittag - -pause \\
\hline Oturma odası & Wohnzimmer & Tarih öğretmeni & Geschicht-s-lehrer \\
\hline Uçak bileti & Flugticket & Çocuk doktoru & Kind - er-arzt \\
\hline & & Mercimek çorbası & Lins $(\boldsymbol{e})$ - n-suppe \\
\hline & Çikolata kutusu & Schokolad(e) - n-schachtel \\
\hline
\end{tabular}

Anstatt der ganzen Übersetzungsaufgabe wurde grundsätzlich die Übersetzung der Komposita berücksichtigt. Komposita wie „Wohnzimmer“ und „Flugticket“ wurden als die meist begegneten, besonders ausgesucht. Wieder „Sommerregen“ kam im letzten Übersetzungsunterricht vom Deutschen ins Türkische im Text vor. Jedoch wurde es als Kompositum nicht vorgestellt.

Tablo 2: Aufgabenleistungen ohne Fugenelemente

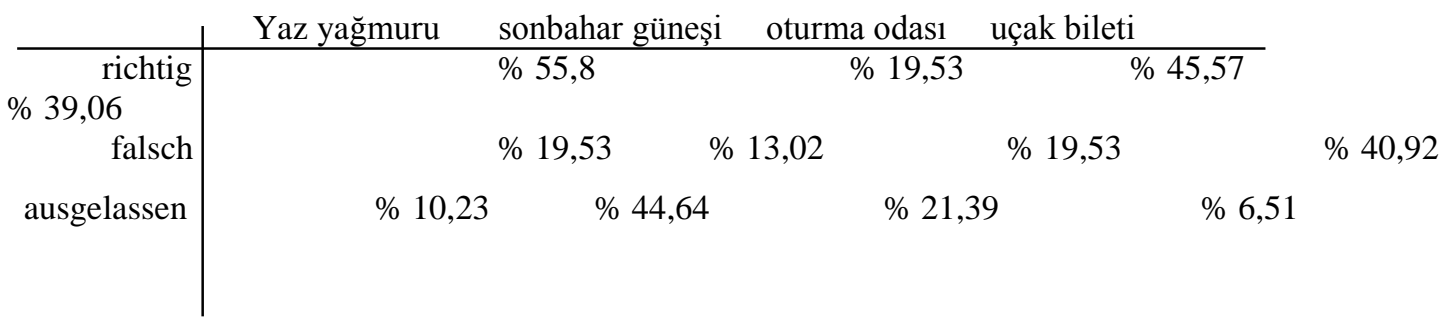

Die Übungsblätter legten dar, dass die Lernenden bei der Übersetzung der unvollständigen Genitive mit denen sie vorher schon begegnet sind erfolgreicher sind. Mit \% 55,8 zeigt das Kompositum Sommerregen die beste Quote. Denn sie sahen, lasen und übersetzten es vor ein paar Wochen im Unterricht. Dennoch ist der Erfolg nicht wie gewünscht. Bei den meisten Übungen fehlt nicht nur ihre Zusammenschreibung, sondern auch die begriffliche Einheit. Die meisten Lernenden schrieben „Sommeregeln/ Sommer Regeln/ Sommerregnerisch /Sommeregnet“" die eigentlich zu einem ganz anderen Begriff führen und ihrer Übersetzung nicht entsprechen. Sie bevorzugen auch andere Konstruktionen außer Kompositionen. Wie in diesen Beispielen:

- Die Regen im Sommer geht früh weg.

- Sommers Regen vergeht.

Die Übersetzung für „Oturma odas““ zeigt keinen großen Unterschied. Obwohl die Studenten diesen Begriff schon in der Vorbereitungsklasse lernen haben sie das Bestimmungswort wörtlich übersetzt (oturmak $=$ setzen). Es sind Übersetzungen wie unten geschrieben worden:

- Alle Hause gibt ein setzen raum es.

- In jedes Haus gibt es ein Sitzenraum.

- Es gibt eine lebenzimmer in alle Häusern.

Trotz diesen nicht äquivalenten Übersetzungen ist der Zusammenschreibungsbewusst zu sehen. Das Kompositum ist 3-mal getrennt geschrieben und 8-malsind Auslassungen vom Bestimmungswort zu beobachten. 
Obwohl der unvollständige Genitiv „sonbahar güneşi/güz güneşi““ im Türkischen sehr oft benutzt wird ist ihre Übersetzung im Deutschen misslungen. Einzeln übersetzt wie sonbahar - Herbst; güneş - Sonne konnte schon eine nahe Entsprechung erlangt werden und wurde auch von 16 Studenten versucht. Aber unter diesen sind auch vollständige Genitiv Versuche zu bemerken.

- Die Sonne des Herbsts schlägt durch die Fenster.

- Die Sonne des Herbst ..... dem Fenster.

- Am Fenster kommt der Herbst der Sonne.

Die Leistungsmessung zeigt, dass die Hälfte der Schüler wohl mit der Komposition „Herbstsonne“ seltener begegnet ist. Denn sie ist zu \%44,64 ausgelassen worden. Die richtige Bildung der Komposition deutet einen Durchschnitt von\% 19,53 an. Wogegen mit \%13,02 der Fehlgriff zu bestimmen ist. Dagegen könnte dieser Fehler durch eine kleine Kenntnis über der Bildung von Komposita beiseitegelegt werden.

Der unvollständige Genitiv „uçuş bileti““ wurde als „Flugticket“ „Flugkarte“ richtig akzeptiert. Denn „ticket“ ist zwar Englisch aber in der Deutschen Alltagsprache gängig. Trotzdem war die Erfolgsquote bei richtig und falsch fast gleich. Weil bei den meisten Übersetzungen wieder entweder das Verb „fliegen“ benutzt oder die Begriffe getrennt geschrieben wurden. Wieder ist die erwartete Leistung nicht eingetroffen.

- Ich nehme zwei fliegen Ticket in Istanbul.

- Ich kaufe zwei Flugzeug Ticket für Istanbul.

- Ich habe zwei Fliegenticket für İstanbul gekauft.

- Ich kaufe zwei Fahrkarte um Istanbul zu fliegen.

Tablo 3: Aufgabenleistungen mit Fugenelemente

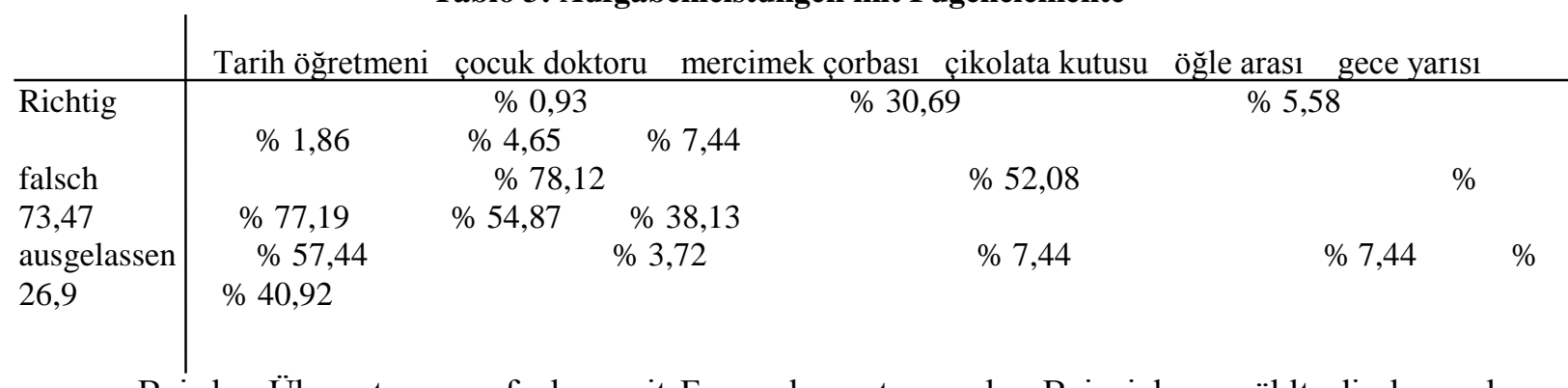

Bei den Übersetzungsaufgaben mit Fugenelemente wurden Beispiele gewählt, die besonders eine der Fugenelemente -s, -en, und -er implizieren. Denn in Komposita kommen am meisten diese Fugen vor. Doch bei ihrer Bildung war die Durchfallquote, wodurch die Ahnungslosigkeit der Lernenden leicht festzustellen war, sehr hoch. Wieder wurden die meisten Wörter getrennt und ohne Fugenelement geschrieben.

Die Übersetzung von dem unvollständigen Genitiv „,tarih öğretmeni“ fordert für ihre deutsche Übertragung die Zusammensetzung von Geschichte und Lehrer mit dem Fugenelement $-s$. Es soll mit diesem Bindemittel als Geschichtslehrer zusammengeschrieben werden. Doch nur \% 0,93 der Lernenden haben die Komposita richtig geschrieben. Die meisten der Übungen waren wieder getrennt und ohne Fugenelement übersetzt. Wiederum waren auch Genitivübersetzungen und falsche Begriffsübertragungen zu sehen.

- Der Geschichte Lehrer hat den II. Weltkrieg erzählt. 
- Der Geschichtelehrer hat II. Weltkrieg erzählt.

- Der Historische Lehrer hat die zweite Welt Krieg gesagt.

- Lehrer der Geschichte erläuterte „2. Weltkrieg“.

Obwohl die Komposition „Kinderarzt“ besonders bei dem Kapitel „Berufe“ vorkommt und den Lernenden eigentlich nicht fremd ist machen die Lernenden große Übersetzungsfehler. Zwar steigt hier die Quote der richtigen Antworten bis zu \%30,69. Aber die falschen Beantwortungen stehen mit \% $52,08 v i e l$ höher. Entweder schreiben sie die Komposition mit dem richtigen Fugenelement aber getrennt (Kinder Arzt), oder ohne Fugenelement zusammen (Kindarzt). Jedoch haben die Studenten bei dieser Übung in einer Mehrzahl die Komposita Zusammengeschrieben aber meistens ohne das Fugenelement - er. Es sind auch Übersetzungen mit Relativ- oder Genitivbildung zu beobachten.

- Das Hospitale hat kein Kinder Arzt.

- In diesem Krankenhaus gibt es keinen Kindarzt.

- In dieser Krankenhausgibt kein die Arzt des Kind.

- In diesem Krankenhaus gibt es keinen Arzt, der sich mit Kindern beschäftigen soll.

- In diesem Krankenhaus gibt es kein Arzt, der mit Kinder kümmert.

Genauso sind die Übersetzungen von dem unvollständigen Genitiv „Mercimek çorbası“ misslungen. Nur \%5,58 der Studenten haben die erwünschte Antwort gegeben. Der Fehlschlag mit einer Quote von \% 73,47 zeigt, dass die Lernenden mit dem Begriff „Linsensuppe“ überhaupt nicht entgegengekommen sind. Zwar schreiben 17 Studenten „Linse“ und „Suppe“ zusammen jedoch ohne das Fugenelement $-\mathrm{n}$. Die anderen schreiben sie entweder getrennt oder ohne das Bestimmungswort.

- Mein Liblingsessen ist die Linse Suppe.

- Meine liebste Essen ist die Linsesuppe.

- Mein Lieblingsessen ist die Suppe.

Bei der Übung „çikolata kutusu“ fehlt auch das Fugenelement und die Zusammenschreibung. Doch noch auffälliger ist das Fehlen der Gegenentsprechung vom Begriff „kutu“. Die meisten Studenten haben es entweder ausgelassen oder nur das Bestimmungswort ,çikolata“ übersetzt und das Grundwort weggelassen. Manche haben es anstatt „Schachtel“ mit „Packung“ oder „Box“ übersetzt, dass teilweise zugelassen werden kann. Dabei fehlen aber wieder das Fugenelement und die Zusammenschreibung.

- Auf meinem Tisch ist eine Packung Schokolade.

- Auf dem Tisch liegt eine Schokolade ...

- Auf dem Tisch legt eine Schokolade Box.

- Auf dem Tisch legt eine Schokolade packet.

Die Komposition Mittagspause ist eigentlich ein sehr bekanntes Wort, dem man in Texten öfters entgegenkommt. Aber auch ihre Übersetzung enttäuscht sehr. Sie zeigt eine Fehlquote von \% 54,87. Bei den meisten Übungen wurde der Begriff entweder einfach ausgelassen oder nur das Bestimmungswort übersetzt. Es sind auch eine Unzahl von Fehlübersetzungen zu sehen. Oft haben sie getrennt/zusammen ohne Fugenelement niedergeschrieben.

- Wir haben mit unseren Freundinnen getroffen.

- Wir treffen sich mit meinen Freunden durch Mittag. 
- Wir treffen mit Freunde Mittelpause.

- Wir treffen die Mittagpause mit unserer Freunde.

- Am Mittag Pause treffen wir mit Freunden.

"Gece yarıs1“ ist auch ein temporales Adverb das durch die Verbindung mit dem Fugenelement - er als „Mitternacht“ wiedergegeben sollte. Doch diese Übung ist die erfolgsloseste Aufgabe. Sie zeigt einen Durchfall mit \%38,13 und Auslassung von \% 40,92. Nur 8 Lernende haben die richtige Rechtschreibung erzielt. Zwischen den erfüllten Aufgaben sind Übersetzungen in denen das Bestimmungswort fehlt zu sehen oder wieder Alternative in denen das Fugenelement fehlt. Wieder die Genitivbildung ist nicht außer Acht zu lassen und erstaunlicherweise sind auch Fehlübertragungen der Begriffe festzustellen.

- In der Nacht klingelt die Tür.

- Mitten in der Nacht klang die Tür.

- In Nachtmittel knopfelt die Tür.

- Im Halbnacht hat die Tür geklingelt.

- In der Mitte der Nacht wurde die Tür geklungen.

\section{Schlussfolgerung:}

Mit dieser Arbeit wurde versucht festzustellen, wie die DaF Lernenden unvollständige Genitivkonstruktionen des Türkischen, die sich für die Zusammensetzung eignen, ins Deutsche übersetzen und welche Fehlübersetzungen sie dabei machen. Es wurde gesehen, dass sie kenntnislos über die Zusammenschreibung der Nominalkomposita sind und diese Gründe sie von der Zusammenschreibung der Nominalkomposita abhalten.

- Da sie ihrer Muttersprache her der Zusammensetzung fremd sind bevorzugen die DaFLerner grundsätzlich das getrennte Schreibender einzelnen Nomina. Anstatt Nominalkomposita ziehen sie entweder den vollständigen Genitiv oder lange hintereinander gereihte Wörter oder Phrasen vor.

- Weil sie keine Ahnung über den Wortbildungsprozess des Sprachpaares haben können sie die Bildung der Komposita nicht gegenüberstellen.

- Sie schenken der Nominalkomposita im Türkischen eine Angehörigkeit, indem sie einen Possessiv Genitiv bilden aber eigentlich eine grammatische und semantische Fehlübertragung machen.

- Sie haben ausgerechnet keine Kenntnis über Fugenelemente, die die Naht der deutschen Nominalkomposita leisten. Deswegen schreiben sie die unvollständigen Genitive außer die ihnen schon sehr bekannt ist, öfters getrennt, wenn schon zusammen ohne Fugenelemente.

- Auch die Bedeutung dieser Komposita für den deutschen Wortschatz ist ihnen nicht bekannt.

Da ein Drittel der deutschen Sprache aus Nominalkomposita entsteht konnte dieser Bewusst in der Zielsprache ihrer Ausdrucksweise und Wortschatzkenntnis weitgehend beitragen. Deswegen sollte die Übersetzungsmöglichkeit dieser Konstruktionen als Nominalkomposita im Deutschen den DaFLernern unbedingt beigebracht werden. Denn „Wortbildungskenntnisse fördern den schöpferischen Umgang mit eigenen Wissensbeständen und können in verschiedenen Phasen des Lernens in verschiedenen Seminaren (z. B. Übersetzung, Grammatik, Methodik und Didaktik) eingesetzt werden (Ceylan, 2018, s. 200)“.Egal in welchem Unterricht sollten die deutschen und türkischen Wortbildungsprozesse kontrastiv gegenübergestellt und den DaF- Lernenden mitgeteilt werden. Somit kann besonders eine bessere grammatische Beherrschung und flüssigere Anwendung von Deutsch erreicht werden. 


\section{LITERATURVERZEICHNIS}

Balc1, T. (2015). Die Erweiterung des Wortschatzes: Eine Analyse am Beispiel Türkisch - Deutsch. C. A. Ahmet Sarı içinde, Germanistik'e Katkılar. Beiträge zur Germanistik (s. 73 - 82). Ankara: Çizgi Kitapevi.

Balc1, T. (2009). Grundzüge der Türkisch - Deutschen Kontrastiven Grammatik. Adana: Ulusoy Matbas1.

Ceylan, y. (2018). Wortbildung im Türkischen und Deutschen: Eine kontrastiv linguistische Untersuchung der Wortbildung mit Wortbildungsanalysen von DaF Studierenden. Turkish Studies , 185 - 202.

Duden Fremdwörterbuch. (1982). Mannheim: Dudenverlag.

Duden Grammatik der deutschen Gegenwartssprache. (1995). Mannheim: Duden Verlag.

Elshorbagy, F. (2007). Fugenelemente der deutschen Komposita . Düsseldorf.

Ergin, M. (2009). Türk Dil Bilgisi. İstanbul: Bayrak.

Fleischer, W., \& Barz, I. (2012). Wortbildung der deutschen Gegenwartssprache. Berlin: De Gruyter.

Graefen, G., \& Liedke, M. (2012). Germanistische Sprachwissenschaft. A. Franke Verlag : Tübingen.

Kahramantürk, K. (1998). Nominale Wortbildung und Nominalisierungen im Deutschen und im Türkischen. Mannheim: Julius Groos Verlag.

Korkmaz, Z. (2009). Türkiye Türkçesi Grameri Şekil Bilgisi. Ankara: Türk Dil Kurumu Yayınları.

Özkan, A. (2018). Bir Söz Dizimi Terimi: Özel İsim Grubu. Selçuk Üniversitesi Türkiyat Araştırmaları Dergisi, 1-11.

Pörings, R., \& Schmitz, U. (2003). Sprache und Sprachwissenschaft: eine kognitiv orientierte Einführung. Tübingen: Gunter Narr Verlag.

Röhrborn, K. (1990). Der Begriff des Nominalkompositums in der türkeitürkischen Sprachwissenschaft. Zeitschrift der Deutschen Morgenländischen , 51 - 67.

Schlücker, B. (2012). Die deutsche Kompositionsfreudigkeit Übersicht und Einführung. L. G. Schlücker içinde, Das Deutsche als kompositionsfreudige Sprache.Strukturelle Eigenschaften und systembezogene Aspekte. . Berlin, New York: De Gruyter: De Gruyter.

Uslu, Z. (2016). Türkçe- Almanca Karşılaştırmalı Temel Dilbilgisi. Ankara: Anı Yayıncılık.

Varsami, J. (2008, sommersemester). Fugenelemente im Deutschen. 03 29, 2019 tarihinde Academia: https://www.academia.edu/11618988/Fugenelemente_im_Deutschen adresinden alınd1

Weber, A. K. (2016). Von Bonsaihund, Runzelmaulwurf und Monchichi-Bärchen Eine Studie zu Adhoc-Nominalkomposita des Deutschen in der Translation anhand deutscher, französischer und italienischer Ausgangs- und Zieltexte. Saarbrücken: universaar.

Wegener, H. (2006). Entstehung und Funktion der Fugenelemente im Deutschen, oder: warum wir keine Autosbahn haben. Linguistische Berichte , 425-457.

Zülfikar, H. (2013). Yazım Kılavuzlarında Kelimeleri Bitişik veya Ayrı Yazma Sorunu. Türk Dili Dil ve Edebiyat Dergisi , 29. 